\title{
CASIMIR FRICTION FORCE AND ENERGY DISSIPATION FOR MOVING HARMONIC OSCILLATORS
}

\author{
Johan S. Høy® \\ Department of Physics, Norwegian University of Science and Technology, \\ N-7491 Trondheim, Norway \\ Iver Brevil2 2 \\ Department of Energy and Process Engineering, Norwegian University of \\ Science and Technology, N-7491 Trondheim, Norway
}

\begin{abstract}
The Casimir friction problem for a pair of dielectric particles in relative motion is analyzed, utilizing a microscopic model in which we start from statistical mechanics for harmonically oscillating particles at finite temperature moving nonrelativistically with constant velocity. The use of statistical mechanics in this context has in our opinion some definite advantages, in comparison with the more conventional quantum electrodynamic description of media that involves the use of a refractive index. The statistical-mechanical description is physical and direct, and the oscillator model, in spite of its simplicity, is nevertheless able to elucidate the essentials of the Casimir friction. As is known, there are diverging opinions about this kind of friction in the literature. Our treatment elaborates upon, and extends, an earlier theory presented by us back in 1992. There we found a finite friction force at any finite temperature, whereas at zero temperature the model led to a zero force. As an additional development in the present paper we evaluate the energy dissipation making use of an exponential cutoff truncating the relative motion of the oscillators. For the dissipation we also establish a general expression that is not limited to the simple oscillator model.
\end{abstract}

PACS numbers: 05.40.-a, 05.20.-y, 34.20.Gj

\footnotetext{
${ }^{1}$ johan.hoye@ntnu.no

2iver.h.brevik@ntnu.no
} 


\section{Introduction}

The Casimir effect - the original paper being Ref. [1] - has attracted a formidable increase of interest in the later years. For instance, as shown by a graph in the 2005 Lamoreaux paper [2], the number of citations per year to the 1948 Casimir paper has increased from about 10 to well above 100 in the period from 1980 to 2010.

The standard Casimir setup is that of two parallel metallic or dielectric plates at micron or sub-micron separations, the attractive force between them being calculated or measured. Later years have seen a considerable progress in the analysis of Casimir forces also between bodies of a more general shape, under static conditions. Some recent general treatments can be found in Refs. [3, 4, 5, 6].

A special sub-class of Casimir phenomena is that of friction. The magnitude of the Casimir friction force between plane surfaces in relative parallel motion has attracted considerable interest recently. The problem as such is not new; it was studied a long time ago by Teodorovich [7] and by Levitov [8]. Polarization currents fluctuating in the two bodies interact via the electromagnetic field, transferring photons between the slabs. Because of the relative motion the photons are subject to Doppler shifts, and one should expect there to be a resulting dissipation of energy, meaning a friction force. The works mentioned were based upon macroscopic theory of the electromagnetic field in a medium, involving use of a refractive index. Most of the more recent literature has been formulated along the same macroscopic lines. Some of the later papers can be found in Refs. [9, 10, 11, 12, 13, 14, 15, 16, 17, 18].

A complicating factor in the macroscopic theory is that there exists no natural rest inertial frame wherein the system of bodies is collectively at rest. This may be a major reason why diverging results are found in the current literature. For instance, Philbin and Leonhardt find there to be no quantum friction at all [13, whereas most other papers find the friction force to be nonvanishing. Recent papers of Dedkov and Kyasov [17] and of Pendry 18 give useful overviews and comparison with earlier results. The title of Pendry's paper [18] is illustrative for this kind of research: "Quantum friction - fact or fiction?".

Faced with this rather complex situation it becomes natural to inquire to what extent light can be shed on the problem by following an alternative approach: Instead of starting from quantum electrodynamics in a medium one can start with a simpler microscopic model in which only moving har- 
monic oscillators are involved. Such an investigation is the main theme of the present paper. We shall consider two oscillators in relative motion having a constant nonrelativistic velocity. They are taken to represent a pair of polarizable particles. As we shall see, this oscillator model is in fact able to describe the essentials of the Casimir friction force. Some years ago we investigated such a microscopic model, for instantaneous [19], as well as for non-instantaneous [20], interactions. In view of the mentioned diverging results obtained in this area of research, we find reason to consider this simple model anew, and to elaborate on it, the more so since results obtained in the model in essence should be valid for dielectric plates in relative motion also. The statistical mechanical approach implies that the Kubo formula [21, 22 ] will play an important role. This way of approaching the Casimir friction problem is apparently not so well known in the Casimir community, but we find it right to emphasize the microscopic method's usefulness. It leads to physical results without use of a heavy mathematical formalism. The microscopic approach has been followed in a recent investigation by Barton also (personal communication).

We assume in the following thermal equilibrium conditions, and assume the interaction between the oscillators to be weak.

The results of the present paper can be summarized as follows:

- There is a finite Casimir force, at any finite temperature $T$.

- For the simple oscillator model the Casimir force vanishes at $T=0$.

- The Casimir energy dissipation is calculated, identifying the dissipation with the work done. Assuming infinite motion (i.e., the same velocity for all times), it is not clear how to distinguish between reversible energy change and irreversible energy dissipation. We handle this problem by introducing a convergence factor by means of which the interaction is limited to a finite time interval. Therewith the energy dissipation is calculated unambiguously.

- The final expression for the energy dissipation, Eq. (27) below, has a validity beyond the limitations of the harmonic oscillator model. Since this is a new result, it would be of interest to have it verified also in other ways, if possible.

We mention finally that techniques similar to those used in the present letter have been used, for instance, in studies of the dynamical Casimir force associated with longitudinal motion of the plates (thus different from the lateral motion considered here). More information can be found in review 
articles [23, 24], with further references therein.

\section{Calculation of the friction force}

We will consider the quantum mechanical two-oscillator system whose reference state is the one of uncoupled motion corresponding to a Hamiltonian $H_{0}$. The equilibrium situation becomes perturbed by a time dependent term which we will write in general form as $-A q(t)$, where $A$ is a time independent operator and $q(t)$ a classical function of time whose explicit form depends on the specific properties of the system. The Hamiltonian becomes $H=H_{0}-A q(t)$. Moreover, we put

$$
-A q(t)=\psi(\mathbf{r}(t)) x_{1} x_{2}
$$

where $\psi(\mathbf{r})$ is the so-called coupling strength (i.e. $\psi$ is the classical potential between the oscillators). The separation between the oscillators is $\mathbf{r}$, and $x_{1}, x_{2}$ are the internal vibrational coordinates of the oscillators. When the oscillators move with respect to each other the coupling has to vary in time. With nonrelativistic constant relative velocity $\mathbf{v}$ the interaction will vary as

$$
-A q(t)=\left[\psi\left(\mathbf{r}_{0}\right)+\nabla \psi\left(\mathbf{r}_{0}\right) \cdot \mathbf{v} t+\ldots\right] x_{1} x_{2},
$$

when expanded around the initial position $\mathbf{r}=\mathbf{r}_{0}$ at $t=0$. The force between the oscillators, called $\mathbf{B}$, is

$$
\mathbf{B}=-(\nabla \psi(\mathbf{r})) x_{1} x_{2}
$$

We ought here to mention the following point. In a mathematical sense the expansion (2) requires $v t$ to be small. Physically, we assume nevertheless Eq. (2) to hold for all times, so that the interaction energy is taken to be proportional to $t$ for all values of $t$. The natural opportunity of choosing $q(t)=t$ in the interaction (11) thus has to be modified: as will be shown below, a convergence factor will be needed.

Another point worth noticing is that the expression (11) corresponds to first quantization only. Quantum electrodynamic processes such as emission and absorption of photons (second quantization) are not accounted for by the present model. They were considered, however, in Ref. [20].

The equilibrium situation with both oscillators at rest is represented by the first term in (2). It gives rise to a (reversible) equilibrium force. Thus 
the friction must be connected with the second term. To simplify, we will for the moment neglect the first term. By this the two oscillators will be fully uncorrelated in their relative position $\mathbf{r}=\mathbf{r}_{0}$. The friction force, due to the time dependence of the interaction (2), will be a small perturbation upon the equilibrium situation. This interaction leads to a response $\Delta\langle\mathbf{B}(t)\rangle$ in the thermal average of $\mathbf{B}$. And this is where the Kubo formula, mentioned above, comes in [21, 22, 25]:

$$
\Delta\langle\mathbf{B}(t)\rangle=\int_{-\infty}^{t} \phi_{B A}\left(t-t^{\prime}\right) q\left(t^{\prime}\right) d t^{\prime}
$$

(note that $\phi_{B A}$ means a vector), where the response function is given by

$$
\phi_{B A}(t)=\frac{1}{i \hbar} \operatorname{Tr}\{\rho[\mathrm{A}, \mathrm{B}(\mathrm{t})]\} \text {. }
$$

Here $\rho$ is the density matrix and $\mathbf{B}(t)$ is the Heisenberg operator $\mathbf{B}(t)=$ $e^{i t H / \hbar} \mathbf{B} e^{-i t H / \hbar}$, where $\mathbf{B}$ like $A$ is time independent. Now with (2) and (3), and with $q(t)=t$, expression (5) can be rewritten as

$$
\phi_{B A}(t)=\mathbf{G} \phi(t)
$$

with

$$
\begin{gathered}
\mathbf{G}=(\nabla \psi)(\mathbf{v} \cdot \nabla \psi), \\
\phi(t)=\operatorname{Tr}\{\rho \mathrm{C}(\mathrm{t})\}, \\
C(t)=\frac{1}{i \hbar}\left[x_{1} x_{2}, x_{1}(t) x_{2}(t)\right] .
\end{gathered}
$$

Thus with Eq. (4) and $q\left(t^{\prime}\right)=t^{\prime}$ the force can be written as

$$
\mathbf{F}=\Delta\langle\mathbf{B}(t)\rangle=\mathbf{G} \int_{-\infty}^{t} \phi\left(t-t^{\prime}\right) t^{\prime} d t^{\prime}=\mathbf{F}_{r}+\mathbf{F}_{f},
$$

where

$$
\mathbf{F}_{r}=\mathbf{G} t \int_{0}^{\infty} \phi(u) d u
$$

is part of the reversible force by which the part of the force that represents friction is

$$
\mathbf{F}_{f}=-\mathbf{G} \int_{0}^{\infty} \phi(u) u d u .
$$

Here the new variable $u=t-t^{\prime}$ has been introduced. The $\mathbf{F}_{r}$ can be interpreted as a reversible force since it depends only upon position. This 
interpretation is consistent with the result obtained for the dissipation in Sec. 3 below; the $\mathbf{F}_{r}$ will not contribute to the net total dissipation.

If one again includes $\psi\left(\mathbf{r}_{0}\right)$ one has

$$
\begin{gathered}
\mathbf{G} t=(\nabla \psi)(\mathbf{v} \cdot \nabla \psi) t \rightarrow(\nabla \psi)\left[\psi\left(\mathbf{r}_{0}\right)+t \mathbf{v} \cdot \nabla \psi+\ldots\right] \\
=(\nabla \psi) \psi\left(\mathbf{r}_{0}+\mathbf{v} t\right)
\end{gathered}
$$

where $\mathbf{r}=\mathbf{r}_{0}+\mathbf{v} t$ is the position at time $t$. By contrast, expression (9) changes sign when the velocity $\mathbf{v}$ changes sign, and it thus represents a friction force. (Observe that the velocity in (10) merely represents the shift in position.) Equation (9) is the same as result (2.11) in Ref. [19] 3, and the Fourier transformed version of it and Eq. (9) above as well, is

$$
\mathbf{F}_{f}=-\left.i \mathbf{G} \frac{\partial \tilde{\phi}(\omega)}{\partial \omega}\right|_{\omega=0},
$$

where $\tilde{\phi}(\omega)=\int_{0}^{\infty} \phi(t) e^{-i \omega t} d t \quad($ with $\phi(t)=0$ for $t<0)$.

In Ref. [19] the Fourier transformed version (11) was used to obtain the explicit expression for the friction force. Here, we instead will use a different approach based on the expression (9). As in the reference mentioned we then need the commutator (77). This entity again follows from the properties of quantized harmonic oscillators. We introduce annihilation and creation operators $a$ and $a^{\dagger}$ with commutation relations $\left[a_{i}, a_{i}^{\dagger}\right]=1(i=1,2$; other commutators vanish). As usual, $a_{j}(t)=a_{j} e^{-i \omega_{j} t}$ and $a_{j}^{\dagger}(t)=a_{j}^{\dagger} e^{i \omega_{j} t}$. With this the coordinates are

$$
x_{i}=\left(\frac{\hbar}{2 m_{i} \omega_{i}}\right)^{1 / 2}\left(a_{i}+a_{i}^{\dagger}\right)
$$

where $m_{i}$ and $\omega_{i}(i=1,2)$ are the mass and eigenfrequency of each oscillator. To obtain $\phi(t)$ from (7) we first have to calculate $\left\langle n_{1} n_{2}|C(t)| n_{1} n_{2}\right\rangle$, where $\left|n_{1} n_{2}\right\rangle=\left|n_{1}\right\rangle\left|n_{2}\right\rangle$ represents eigenstates with oscillators excited to levels $n_{1}$ and $n_{2}$. One has, when taking into account standard properties of the annihilation and creation operators,

$$
L_{i} \equiv\left\langle n_{i}\left|a_{i}^{\dagger} a_{i}(t)+a_{i} a_{i}^{\dagger}(t)\right| n_{i}\right\rangle=\left(2 n_{i}+1\right) \cos \left(\omega_{i} t\right)+i \sin \left(\omega_{i} t\right),
$$

\footnotetext{
${ }^{3}$ There is a missing minus sign in Eq. (2.11) and the $\mathbf{F}_{r}$ was not taken into account.
} 
from which the thermal average follows after some computations as

$$
\begin{gathered}
\phi(t)=\left\langle\left\langle n_{1} n_{2}|C(t)| n_{1} n_{2}\right\rangle\right\rangle \\
=\frac{1}{i \hbar} \frac{\hbar}{2 m_{1} \omega_{1}} \frac{\hbar}{2 m_{2} \omega_{2}}\left(L_{1} L_{2}-L_{1}^{*} L_{2}^{*}\right. \\
=D\left[\left(2\left\langle n_{1}\right\rangle+1\right) \cos \left(\omega_{1} t\right) \sin \left(\omega_{2} t\right)+\left(2\left\langle n_{2}\right\rangle+1\right) \cos \left(\omega_{2} t\right) \sin \left(\omega_{1} t\right)\right] .
\end{gathered}
$$

Here

$$
D=\frac{\hbar}{2 m_{1} m_{2} \omega_{1} \omega_{2}} .
$$

With energy levels $\varepsilon_{n}=\left(n+\frac{1}{2}\right) \hbar \omega$ the thermal average for the occupation numbers is

$$
2\left\langle n_{i}\right\rangle+1=\operatorname{coth}\left(\frac{1}{2} \beta \hbar \omega\right) .
$$

In Ref. [19] expression (14) was Fourier transformed to obtain the friction force as given by (11). As an alternative we will here use expression (14) directly in Eq. (9). Then we get the integral

$$
\begin{gathered}
\int_{0}^{\infty} t e^{-\eta t} \cos \left(\omega_{1} t\right) \sin \left(\omega_{2} t\right) d t \\
=\frac{\eta \Omega_{1}}{\left(\eta^{2}+\Omega_{1}^{2}\right)^{2}}-\frac{\eta \Omega_{2}}{\left(\eta^{2}+\Omega_{2}^{2}\right)^{2}} \rightarrow-\frac{\pi}{2 \Omega_{2}} \delta\left(\Omega_{2}\right), \quad \eta \rightarrow 0 .
\end{gathered}
$$

Here $\Omega_{1}=\omega_{1}+\omega_{2}$ and $\Omega_{2}=\omega_{1}-\omega_{2}$. As mentioned above a convergence factor $e^{-\eta t}$ is needed, and the limit $\eta \rightarrow 0$ is taken. Then the $\Omega_{2}$-term becomes a delta function with prefactor determined by the integral

$$
\int_{-\infty}^{\infty} \frac{\eta x^{2}}{\left(\eta^{2}+x^{2}\right)^{2}} d x=\frac{\pi}{2}
$$

From (14) we also get this integral with $\omega_{1}$ and $\omega_{2}$ interchanged. This will then give the result (16) with opposite sign with respect to the $\Omega_{2}$-term. Adding up we get the difference of the prefactors

$$
\operatorname{coth}\left(\frac{1}{2} \beta \hbar \omega_{1}\right)-\operatorname{coth}\left(\frac{1}{2} \beta \hbar \omega_{2}\right)=-\frac{\sinh \left(\frac{1}{2} \beta \hbar \Omega_{2}\right)}{\sinh \left(\frac{1}{2} \beta \hbar \omega_{1}\right) \sinh \left(\frac{1}{2} \beta \hbar \omega_{2}\right)}
$$




$$
\rightarrow-\frac{\frac{1}{2} \beta \hbar \Omega_{2}}{\sinh \left(\frac{1}{2} \beta \hbar \omega_{1}\right) \sinh \left(\frac{1}{2} \beta \hbar \omega_{2}\right)}, \quad \eta \rightarrow 0 .
$$

Multiplying (16) with (18) and including the factors $D$ and $\mathbf{G}$ the friction force becomes

$$
\mathbf{F}_{f}=-\frac{\pi \beta \hbar^{2}(\nabla \psi)(\mathbf{v} \cdot \nabla \psi)}{8 m_{1} m_{2} \omega_{1}^{2} \sinh ^{2}\left(\frac{1}{2} \beta \hbar \omega_{1}\right)} \delta\left(\omega_{1}-\omega_{2}\right),
$$

which is also the result (3.14) of Ref. [19]. Again one notes that there is friction only when the oscillators have the same frequency, and $\beta$ should be finite, i.e. $T>0$.

When $\beta \rightarrow \infty$, the expression (19) vanishes. According to the present oscillator model there is thus no friction force at zero temperature. An objection against this result may be that it is somewhat singular due to the presence of the $\delta$-function. Thus its physical significance may not be obvious. However, $\eta$ can be kept finite. This will smooth out the $\delta$-function, and the $\Omega_{1}$-term in Eq. (16) will give a contribution too. Note that this will not change our conclusions about a finite friction force for $T>0$. (For finite $\eta$, i.e. interaction like a short pulse, there will also be a contribution for $T=0$ due to the $\Omega_{1}$-term in Eq. (16).) But here we will assume $\eta$ small by which the $T=0$ contribution can be disregarded.

In Ref. [19] the result (19) for the friction force was derived also by two other methods. These methods utilized the path integral formalism of quantum systems at thermal equilibrium [26]. The path integral can be identified with a classical polymer problem where imaginary time is a fourth dimension of length $\beta$. Thus the polymers stretch out in the fourth dimension and form closed loops of periodicity $\beta$. For harmonic oscillators the correlation function along the polymers is obtained in a straightforward way. With one of the methods the convolution of the correlation functions of both oscillators were needed. The resulting Fourier transform of this convolution was then identified with the response function $\tilde{\phi}(\omega)$ used in expression (11) [25].

With the other method full thermal equilibrium was utilized. Then the relative motion of the oscillators was regarded as a harmonic oscillator motion with low frequency $\omega_{0} \rightarrow 0$. Again with the path integral one can obtain the Fourier transform of the response function for the relative motion. The damping of the relative motion, that can be related to this response function, gives the friction force, and again the result (19) was recovered. Thus the three methods used in Ref. [19], as well as the modification considered in 
the present paper, all lead to the same result, in contradiction to some other results in the literature, for instance that of Ref. [13].

The result for the friction force was also extended to the situation with time-dependent or non-instantaneous interaction [20]. Then the full thermal equilibrium method was applicable to generalize the result. With the latter interaction there was also a friction from the self-interaction of a moving oscillator with itself.

\section{Dissipation of energy}

The presence of friction means dissipation of energy; the thermal energy of the system has to increase. However, this increase will be of second order in the velocity. As the Kubo formalism used above is limited to linear response, it is not immediately obvious how to evaluate the increase in energy or change in the Hamiltonian $H$. Simply replacing the operator $B$ above with $H$ will not work. However, for the present model it is possible to identify the dissipation directly with the work done. Work per unit time is force times velocity. During the perturbation period it is not obvious or possible how to distinguish between reversible change of energy and irreversible dissipation of energy. But this problem can be circumvented by considering the total energy change due to a perturbation that lasts for a finite time interval. This is the method that we will use in the following.

Thus assume that the relative motion is finite in time, and that it starts at $t=0$ with maximum velocity $\mathbf{v}$ when the position is $\mathbf{r}=\mathbf{r}_{0}$. As $t \rightarrow \infty$ the motion is required to die out. To accomplish this we introduce the convergence factor $e^{-\eta t}(\eta \rightarrow 0)$ already used in Eq. (16). In the interaction (2), $t$ is thus to be replaced with $q(t)=t e^{-\eta t}$. As mentioned we consider only the time interval $0 \leq t<\infty$, in which the velocity decays exponentially,

$$
\mathbf{v} \rightarrow \mathbf{v}_{1}(t)=\mathbf{v} \dot{q}(t)=\mathbf{v}(1-\eta t) e^{-\eta t}
$$

For $\eta t>0, \mathbf{v}_{1}(t)$ will now replace $\mathbf{v}$ in expression (19) for the friction force. Altogether, the total energy dissipated will be

$$
\Delta E_{d}=\int_{-\infty}^{\infty} \mathbf{v}_{1}(t) \cdot \mathbf{F}_{f} \dot{q}(t) d t=\mathbf{v} \cdot \mathbf{F}_{f} \int_{0}^{\infty}[\dot{q}(t)]^{2} d t=\frac{1}{4 \eta} \mathbf{v} \cdot \mathbf{F}_{f},
$$

where $\mathbf{F}_{f}$ is given by Eq. (19). Note that the reversible part of the force $\mathbf{F}_{r} \propto t \rightarrow q(t)$ as given by Eq. (8) will not contribute to the dissipation since $\int_{0}^{\infty} \dot{q}(t) q(t) d t=0$. 
The above result for dissipation may be extended to more general cases for which $q(t)$ shows an arbitrary variation with time. Thus $q(t)$ need no longer be linked to a varying position in space as shown in Eq. (1). Under quite general conditions we may reinterpret the situation as one for which $q(t)$ is associated with a "position",

$$
x(t)=q(t)
$$

With this the "velocity" becomes

$$
v(t)=\dot{q}(t)
$$

As $x(t)$ is a scalar quantity the corresponding operator $B$ for the "force" will be

$$
B=-\frac{\partial}{\partial x}(-A q(t))=A
$$

With relation (44) the resulting "force" due to the perturbation becomes

$$
F_{f}=\int_{-\infty}^{t} \phi_{A A}\left(t-t^{\prime}\right) q\left(t^{\prime}\right) d t^{\prime}
$$

where

$$
\phi_{A A}(t)=\frac{1}{i \hbar} \operatorname{Tr}\{\rho[A, A(t)]\} .
$$

With the associations made above the total dissipated energy becomes

$$
\Delta E_{d}=\int_{-\infty}^{\infty} v(t) F_{f} d t=\int_{-\infty}^{\infty} \dot{q}(t)\left[\int_{-\infty}^{t} \phi_{A A}\left(t-t^{\prime}\right) q\left(t^{\prime}\right) d t^{\prime}\right] d t .
$$

The expression (21) is consistent with this more general result. In the integral (21) a reversible part of the force was taken out. This corresponds to writing

$$
q\left(t^{\prime}\right)=q(t)-\dot{q}(t)\left(t-t^{\prime}\right)+\ldots
$$

The first term will not contribute to $\Delta E_{d}$, and we get $(q(t)=0$ for $t<0)$

$$
\Delta E_{d}=-\int_{0}^{\infty} \phi_{A A}(u) u d u \int_{0}^{\infty}[\dot{q}(t)]^{2} d t+\ldots
$$

which is the result (21). This is seen by use of Eqs. (3), (6) and (9). Then with $q(t)=t \rightarrow t e^{-\eta t}$ one has $A=\mathbf{v} \cdot \mathbf{B}$ and thus $\phi_{A A}=\mathbf{v} \cdot \phi_{B A}=\mathbf{v} \cdot \mathbf{G} \phi$, by which $-\int_{0}^{\infty} \phi_{A A} u d u=\mathbf{v} \cdot \mathbf{F}_{f}$. 
The expression (27) is thus the energy dissipation due to a perturbation $-A q(t)$ of the Hamiltonian. It is a general result, not necessarily limited to the simple model given by Eq. (11). Since we used an indirect argument implying the net work done on an equivalent system, it would be of interest

to verify this result by an independent method. The recent approach of Barton (personal communication) is in this context of interest, as he makes use of quantum mechanical perturbation theory to the second order in the interaction strength. If it could be shown that the different approaches lead to the same results for force and dissipation, it would be physically instructive.

\section{Acknowledgment}

I. B. thanks Gabriel Barton for valuable correspondence.

\section{References}

[1] H. B. G. Casimir, Proc. K. Ned. Akad. Wet. 51, 793 (1948).

[2] S. K. Lamoreaux, Rep. Prog. Phys. 68, 201 (2005).

[3] K. A. Milton, The Casimir Effect: Physical Manifestations of the ZeroPoint Energy (World Scientific, Singapore, 2001).

[4] K. A. Milton, J. Phys. A 37, R209 (2004).

[5] S. Y. Buhmann and D.-G. Welsch, Prog. Quant. Electron. 31, 51 (2007).

[6] M. Bordag, G. L. Klimchitskaya, U. Mohideen and V. M. Mostepanenko, Advances in the Casimir Effect (Oxford Science Publ., Oxford, 2009).

[7] E. V. Teodorovich, Proc. R. Soc. Lond. A 362, 71 (1978).

[8] L. S. Levitov, Europhys. Lett. 8, 499 (1989).

[9] J. B. Pendry, J. Phys.: Condens. Matter 9, 10301 (1997).

[10] J. B. Pendry, J. Mod. Optics 45, 2389 (1998).

[11] A. I. Volokitin and B. N. J. Persson, Rev. Mod. Phys. 79, 1291 (2007).

[12] A. I. Volokitin and B. N. J. Persson, Phys. Rev. B 78, 155437 (2008). 
[13] T. G. Philbin and U. Leonhardt, New J. Phys. 11, 033035 (2009).

[14] T. G. Philbin and U. Leonhardt, arXiv:0904.2148 [quant-ph].

[15] G. V. Dedkov and A. A. Kyasov, J. Phys.: Condens. Matter 20, 354006 (2008).

[16] G. V. Dedkov and A. A. Kyasov, Technical Physics 53, 389 (2008) [Zhurnal Tekhn. Fiziki 78, 1 (2008)].

[17] G. V. Dedkov and A. A. Kyasov, Surface Science 604, 562 (2010).

[18] J. B. Pendry, New J. Phys. 12, 033028 (2010).

[19] J. S. Høye and I. Brevik, Physica A 181, 413 (1992).

[20] J. S. Høye and I. Brevik, Physica A 196, 241 (1993).

[21] R. Kubo, in Lectures in Theoretical Physics, Vol. I, Boulder 1958. Edited by W. E. Brittin and L. G. Dunham (Interscience, Newitemkubo59 York, 1959).

[22] L. D. Landau and E. M. Lifshitz, Statistical Physics, 3rd ed. Part 1 (Pergamon Press, Oxford, 1985).

[23] M.-T. Jaekel and S. Reynaud, Reports on Progress in Physics 60, 863 (1997).

[24] D. A. R. Dalvit, P. A. Maia Neto and F. D. Mazzitelli, arXiv:1006.4790; to appear in Lecture Notes in Physics, Volume on Casimir Physics, eds. D. A. R. Dalvit et al.

[25] I. Brevik and J. S. Høye, Physica A 153, 420 (1988).

[26] J. S. Høye and G. Stell, J. Chem. Phys. 75, 5133 (1981). 